\title{
Formulation and Solution of Space-Time Fractional KdV-Burgers Equation
}

\author{
Essam M. Abulwafa, Ahmed M. Elgarayhi, Abeer A. Mahmoud, Ashraf M. Tawfik \\ Theoretical Physics Research Group, Physics Department \\ Faculty of Science, Mansoura University, Mansoura 35516, Egypt \\ E-mail: abulwafa@mans.edu.eg
}

Received: 6 August 2013; revised: 18 November 2013; accepted: 6 December 2013; published online: 17 December 2013

\begin{abstract}
The space-time fractional KdV-Burgers equation has been derived using the semi-inverse method and Agrawal's variational method. The modified Riemann-Liouville definition is used for the fractional differential operators. The derived fractional equation is solved using the fractional sub-equation method.
\end{abstract}

Key words: Fractional Euler-Lagrange equation, space-time fractional KdV-Burgers equation, modified Riemann-Liouville fractional definition, fractional sub-equation method

\section{INTRODUCTION}

All forces in nature are nearly non-conservative: dissipative and/or dispersive forces. Classical mechanics, using integer differential equations, treated conservative forces while the non-integer differential equations can be used to describe the non-conservative forces. Fractional calculus is a field of mathematics that grows out of the traditional definitions of calculus. Fractional calculus has gained importance during the last decades mainly due to its applications in various fields of science and engineering. Some of the areas of present day applications of fractional calculus include fluid flow, rheology, dynamical process in self-similar and porous structures, diffusive transport akin to diffusion, electrical networks, probability and statistics, control theory of dynamical systems, viscoelasticity, electro-chemistry of corrosion, chemical physics, optics, and signal processing, and so on [1-7].

There are different kinds of fractional integration and differentiation operators. The most famous one is the RiemannLiouville definition [8-11], which has been used in various fields of science and engineering successfully, but this definition leads to the result that constant function differentiation is not zero. Caputo put definitions which give zero value for fractional differentiation of constant function, but these definitions require that the function should be smooth and differentiable [8-11]. Recently, Jumarie derived definitions for the fractional integral and derivative called modified Riemann-Liouville [12-15], which are suitable for continuous and nondifferentiable functions and give differentiation of a constant function equal to zero. The modified RiemannLiouville fractional definitions are used effectively in many different problems [16-20].

It was shown that non-integer derivatives in the Lagrangian describe non-conservative forces. Riewe [21, 22] derived a method using a fractional Lagrangian that leads to a fractional Euler-Lagrange equation that is, in some sense, equivalent to the desired equation of motion. Hamilton's equations are derived from the Lagrangian and are equivalent to the Euler-Lagrange equation. Further study of the fractional Euler-Lagrange can be found in the works of Agrawal [23-25]. He presented generalized Euler-Lagrange equations for unconstrained and constrained fractional variational problems. Baleanu and coworkers $[26,27]$ used the fractional Euler-Lagrange equation to model fractional Lagrangian and Hamiltonian formulations. El-Wakil et al derived the time fractional forms of some mathematical-physics equations [28] using Agrawal's variational method [23-25] and used them to describe the electrostatic potential in some plasma systems [29]. 
Several methods have been used to solve fractional differential equations such as: Laplace transformation method, Fourier transformation method, iteration method, and operational method [8-11, 30]. However, most of these methods are suitable for special types of fractional differential equations, namely the linear with constant coefficients. However, some papers deal with the existence and multiplicity of solution of the nonlinear fractional differential equation using techniques of nonlinear analysis such as: Adomian decomposition method [31], homotopy perturbation method [32] and variational iteration method [33].

It is common knowledge that many physical problems (such as non-linear shallow-water waves and wave motion in plasmas) can be described by the KdV-type equations. The KdV-type equations also have applications in quantum field theory, plasma physics and solid-state physics. For example, the kink soliton can be used to calculate energy, momentum flow and topological charge in the quantum field. In order to study the problems of the flow of liquids containing gas bubbles, the fluid flow in elastic tubes, etc., the control equation can be reduced to the so-called KdV-Burgers equation. This equation is equal to the $\mathrm{KdV}$ equation if a viscous dissipation term is added. The KdV-Burgers equation can be thought of as a generalization of the $\mathrm{KdV}$ and Burgers equations. This equation combines nonlinearity, linear dissipation and dispersion terms. This is a well known nonlinear model of viscous elastic medium and is found in many physical phenomena. The Burgers equation is a special case of the $\mathrm{KdV}$-Burgers equation has been found to describe various kinds of phenomena such as a mathematical model of turbulence and the approximate theory of flow through a shock wave traveling in viscous fluid.

In this paper, the space-time fractional KdV-Burgers equation is derived using Agrawal's technique [23-25] and the modified Riemann-Liouville derivative [12-15], and solved by the improved fractional sub-equation method $[34,35]$.

The modified Riemann-Liouville fractional derivative $D_{x}^{\alpha} f(x)$ is defined in the form [12-15]

$$
\begin{gathered}
D_{x}^{\alpha}[f(x)-f(a)]=\frac{1}{\Gamma(-\alpha)} \int_{a}^{x} d \xi f(\xi) /(x-\xi)^{\alpha+1}, \quad \alpha<0, \\
D_{x}^{\alpha} f(x)=\frac{1}{\Gamma(1-\alpha)} \\
\times \frac{d}{d x}\left\{\int_{a}^{x} \frac{d \xi[f(\xi)-f(a)]}{(x-\xi)^{\alpha}}\right\}, 0<\alpha<1, \\
D_{x}^{\alpha} f(x)=\frac{d^{n}}{d x^{n}}\left\{D_{x}^{\alpha-n} f(x)\right\}, \quad n \leq \alpha<n+1, n \geq 1 .
\end{gathered}
$$

Some properties of the modified Riemann-Liouville $(\mathrm{mRL})$ fractional derivative were summarized in [12-15], useful formulae include

$$
D_{x}^{\alpha} C=0, \alpha>0, C \text { is a constant, }
$$

$$
\begin{gathered}
D_{x}^{\alpha} x^{\gamma}=\frac{\Gamma(1+\gamma)}{\Gamma(1+\gamma-\alpha)} x^{\gamma-\alpha}, \gamma>0, \\
D_{x}^{\alpha}[u(x) v(x)]=D_{x}^{\alpha}[u(x)] v(x)+u(x) D_{x}^{\alpha}[v(x)], \\
D_{x}^{\alpha} f(u(x))=D_{x}^{\alpha} u(x)\left(\frac{d f}{d u}\right), \\
=D_{u}^{\alpha} f(u)\left(\frac{d u}{d x}\right)^{\alpha}, \\
\int_{a}^{b}(d x)^{\alpha} u(x) D_{x}^{\alpha} v(x)=\alpha !\left[\left.u(x) v(x)\right|_{a} ^{b}\right. \\
-\int_{a}^{b}(d x)^{\alpha} v(x) D_{x}^{\alpha} u(x), \\
u(x), v(x) \in[a, b], \quad 0<\alpha<1 .
\end{gathered}
$$

$v(x)$ is non-differentiable, $u(x)$ is non-differentiable in (3) and (4a) and differentiable in (4b), and $f(u(x))$ is differentiable in (4a) and non-differentiable in (4b).

\section{SPACE-TIME-FRACTIONAL KDV-BURGERS EQUATION FORMULATION}

The space-time-fractional KdV-Burgers (STFKdV-Burgers) equation in $(1+1)$-dimension can be formulated as follows: The regular KdV-Burgers equation has the form

$$
\begin{gathered}
\frac{\partial}{\partial t} u(x, t)+A u(x, t) \frac{\partial}{\partial x} u(x, t) \\
+B \frac{\partial^{2}}{\partial x^{2}} u(x, t)+C \frac{\partial^{3}}{\partial x^{3}} u(x, t)=0 .
\end{gathered}
$$

Using the potential function $U(x, t)$ where $u(x, t)=$ $U_{x}(x, t)$ gives the potential equation of the regular $\mathrm{KdV}$ Burgers equation in the form

$$
\begin{gathered}
U_{x t}(x, t)+A U_{x}(x, t) U_{x x}(x, t) \\
+B u_{x x}(x, t)+C U_{x x x x}(x, t)=0,
\end{gathered}
$$

where the subscripts denote partial differentiation of the function with respect to the parameter. The Euler-Lagrange equation of the regular KdV-Burgers equation can be derived using the semi-inverse method [36, 37] as follows:

The functional of the potential equation can be represented by

$$
\begin{aligned}
& J(U)=\int_{R} d x \int_{T} d t U(x, t)\left\{c_{1} U_{x t}(x, t)+c_{2} A U_{x}(x, t)\right. \\
& \left.\times U_{x x}(x, t)+c_{3} B u_{x x}(x, t)+c_{4} C U_{x x x x}(x, t)\right\},
\end{aligned}
$$

where $c_{1}, c_{2}, c_{3}$ and $c_{4}$ are constant Lagrangian multipliers. Here $R$ refers to the boundaries of the space domain, while $T$ denotes to the initial and final values of the time. Integrating (8) by parts where $\left.U_{x}\right|_{R}=\left.U_{t}\right|_{T}=\left.U_{x x}\right|_{R}=0, u_{x x}(x, t)$ 
is considered as a fixed function and applying the variation of this functional with respect to $U(x, t)$ lead to

$$
\begin{gathered}
\delta J(U)=\int_{R} d x \int_{T} d t\left\{-c_{1}\left[U_{t}(x, t) \delta U_{x}(x, t)\right.\right. \\
\left.+U_{x}(x, t) \delta U_{t}(x, t)\right]-\frac{3}{2} c_{2} A\left[U_{x}(x, t)\right]^{2} \delta U_{x}(x, t) \\
\left.+c_{3} B u_{x x}(x, t) \delta U(x, t)+2 c_{4} C\left[U_{x x}(x, t) \delta U_{x x}(x, t)\right]\right\} .
\end{gathered}
$$

Integrating by parts using $\left.U_{x}\right|_{R}=\left.U_{t}\right|_{T}=\left.U_{x x}\right|_{R}=0$ and optimizing this variation, $\delta J(U)=0$, give

$$
\begin{gathered}
2 c_{1} U_{x t}(x, t)+3 c_{2} A U_{x}(x, t) U_{x x}(x, t) \\
+c_{3} B u_{x x}(x, t)+2 c_{4} C U_{x x x x}(x, t)=0 .
\end{gathered}
$$

Comparing the above equation with (7) gives constant Lagrangian multipliers as

$$
c_{1}=\frac{1}{2}, \quad c_{2}=\frac{1}{3}, \quad c_{3}=1, \quad c_{4}=\frac{1}{2} .
$$

The functional relation yields directly the Lagrangian of the potential equation as

$$
\begin{aligned}
& L\left(U, U_{t}, U_{x}, U_{x x}\right)=\frac{1}{2} U_{t}(x, t) U_{x}(x, t) \\
+ & \frac{1}{6} A\left[U_{x}(x, t)\right]^{3}-B U(x, t) u_{x x}(x, t)-\frac{1}{2} C\left[U_{x x}(x, t)\right]^{2} .
\end{aligned}
$$

Similarly, the Lagrangian of the space-time-fractional version of the KdV-Burgers equation could be written in the form

$$
\begin{aligned}
& F\left(U, D_{t}^{\alpha} U, D_{x}^{\beta} U, D_{x}^{\beta \beta} U\right)= \\
= & \frac{1}{2} D_{t}^{\alpha} U(x, t) D_{x}^{\beta} U(x, t)+\frac{1}{6} A\left[D_{x}^{\beta} U(x, t)\right]^{3} \\
- & B U(x, t) D_{x}^{\beta \beta} u(x, t)-\frac{1}{2} C\left[D_{x}^{\beta \beta} U(x, t)\right]^{2},
\end{aligned}
$$

where $D_{z}^{\gamma \gamma} f(z)=D_{z}^{\gamma}\left[D_{z}^{\gamma} f(z)\right]$ while the fractional derivative $D_{z}^{\gamma} f(z)$ is the $\mathrm{mRL}$ fractional derivative [12-15] defined by (1). The functional of the STFKdV-Burgers equation takes the form

$$
J_{F}(U)=\int_{R}(d x)^{\beta} \int_{T}(d t)^{\alpha} F\left(U, D_{t}^{\alpha} U, D_{x}^{\beta} U, D_{x}^{\beta \beta} U\right) .
$$

The variation of this functional with respect to $U(x, t)$ leads to

$$
\begin{gathered}
\delta J_{F}(U)=\int_{R}(d x)^{\beta} \int_{T}(d t)^{\alpha}\left\{\left(\frac{\partial F}{\partial U}\right) \delta U+\left(\frac{\partial F}{\partial D_{t}^{\alpha} U}\right)\right. \\
\left.\times \delta D_{t}^{\alpha} U+\left(\frac{\partial F}{\partial D_{x}^{\beta} U}\right) \delta D_{x}^{\beta} U+\left(\frac{\partial F}{\partial D_{x}^{\beta \beta} U}\right) \delta D_{x}^{\beta \beta} U\right\} .
\end{gathered}
$$

Integrating this equation by parts using the definition (5) and optimizing this relation, $\delta J_{F}(U)=0$, the Euler-Lagrange equation for the STFKdV-Burgers equation has the form

$$
\begin{aligned}
& \left(\frac{\partial F}{\partial U}\right)-D_{t}^{\alpha}\left(\frac{\partial F}{\partial D_{t}^{\alpha} U}\right) \\
- & D_{x}^{\beta}\left(\frac{\partial F}{\partial D_{x}^{\beta} U}\right)+D_{x}^{\beta \beta}\left(\frac{\partial F}{\partial D_{x}^{\beta \beta} U}\right)=0,
\end{aligned}
$$

with the constraints that $\left.\delta U\right|_{R}=\left.\delta U\right|_{T}=0$.

Substituting the Lagrange of STFKdV-Burgers (10) into this Euler-Lagrange formula gives

$$
\begin{aligned}
& -B D_{x}^{\beta \beta} u(x, t)-D_{t}^{\alpha}\left[D_{x}^{\beta} U(x, t)\right] \\
& -\frac{1}{2} A D_{x}^{\beta}\left[D_{x}^{\beta} U(x, t)\right]^{2}-C D_{x}^{\beta \beta}\left[D_{x}^{\beta \beta} U(x, t)\right]=0 .
\end{aligned}
$$

Substituting $u(x, t)=D_{x}^{\beta} U(x, t)$ and using formula (3) lead to

$$
\begin{aligned}
& D_{t}^{\alpha} u(x, t)+A u(x, t) D_{x}^{\beta} u(x, t) \\
+ & B D_{x}^{\beta \beta} u(x, t)+C D_{x}^{\beta \beta \beta} u(x, t)=0,
\end{aligned}
$$

which is the space-time-fractional Koreweg-de Vries-Burgers equation.

\section{SPACE-TIME-FRACTIONAL KDV-BURGERS EQUATION SOLUTION}

In this section, the STFKdV-Burgers equation will be solved using a fractional sub-equation method [34, 35].

Considering the traveling wave transformations $u(x, t)=$ $\Phi(\xi), \xi=x+v t,(15)$ can be reduced to the following nonlinear fractional ordinary differential equation (FODE) using relation (4) for the case of $\beta=\alpha$ :

$$
\begin{gathered}
v^{\alpha} D_{\xi}^{\alpha} \Phi(\xi)+A \Phi(\xi) D_{\xi}^{\alpha} \Phi(\xi) \\
+B D_{\xi}^{\alpha \alpha} \Phi(\xi)+C D_{\xi}^{\alpha \alpha \alpha} \Phi(\xi)=0 .
\end{gathered}
$$

The fractional sub-equation method [34, 35] assumes solution of this equation as

$$
\Phi(\xi)=\sum_{k=0}^{n} a_{k} \varphi^{k}(\xi),
$$

where $\varphi(\xi)$ satisfies the following fractional Riccati equation:

$$
D_{\xi}^{\alpha} \varphi(\xi)=\sum_{j=0}^{m} b_{j} \varphi^{j}(\xi),
$$

where $a_{k}, k=0, \ldots, n$ are constant coefficients to be determined later and $b_{j}, j=0, \ldots, m$ are arbitrary coefficients.

Balancing the highest order derivative term and nonlinear term in (16) the value of $n$ can be determined, which has in this problem the value $n=2$.

We suppose that (16) has the following formal solution:

$$
\Phi(\xi)=a_{0}+a_{1} \varphi(\xi)+a_{2}[\varphi(\xi)]^{2},
$$


where $a_{0}, a_{1}$ and $a_{2}$ are constant coefficients to be determined and $\varphi(\xi)$ satisfies the following fractional Riccati equation:

$$
D_{\xi}^{\alpha} \varphi(\xi)=b_{0}+b_{2} \varphi^{2}(\xi)
$$

where $b_{0}$ and $b_{2}$ are arbitrary coefficients.

Using the generalized Exp-function method via MittagLeffler functions, Zhang et al [38] first obtained the following solution of the fractional Riccati equation (20)

$$
\begin{array}{ll}
\varphi_{1}(\xi)=-\sqrt{-b_{0}} \tanh _{\alpha}\left(\sqrt{-b_{0}} \xi\right), & b_{0}<0, b_{2}=1, \\
\varphi_{2}(\xi)=-\sqrt{-b_{0}} \operatorname{coth}_{\alpha}\left(\sqrt{-b_{0}} \xi\right), & b_{0}<0, b_{2}=1, \\
\varphi_{3}(\xi)=\sqrt{b_{0}} \tan _{\alpha}\left(\sqrt{b_{0}} \xi\right), & b_{0}>0, b_{2}=1, \\
\varphi_{4}(\xi)=-\sqrt{b_{0}} \cot _{\alpha}\left(\sqrt{b_{0}} \xi\right), & b_{0}>0, b_{2}=1, \\
\varphi_{5}(\xi)=-\Gamma(1+\alpha) /\left(\xi^{\alpha}+\omega\right), & b_{0}=0, b_{2}=1, \\
\omega=\text { constant }
\end{array}
$$

with generalized hyperbolic and trigonometric functions

$$
\begin{gathered}
\tanh _{\alpha}(x)=\sinh _{\alpha}(x) / \cosh _{\alpha}(x), \\
\operatorname{coth}_{\alpha}(x)=\cosh _{\alpha}(x) / \sinh _{\alpha}(x), \\
\sinh _{\alpha}(x)=\left[E_{\alpha}(x)-E_{\alpha}(-x)\right] / 2, \\
\cosh _{\alpha}(x)=\left[E_{\alpha}(x)+E_{\alpha}(-x)\right] / 2, \\
\tan _{\alpha}(x)=\sin _{\alpha}(x) / \cos _{\alpha}(x), \\
\cot _{\alpha}(x)=\cos _{\alpha}(x) / \sin _{\alpha}(x), \\
\sin _{\alpha}(x)=\left[E_{\alpha}(i x)-E_{\alpha}(-i x)\right] /(2 i), \\
\cos _{\alpha}(x)=\left[E_{\alpha}(i x)+E_{\alpha}(-i x)\right] / 2,
\end{gathered}
$$

where $i=\sqrt{-1}$ and $E_{\alpha}(x)$ is the Mittag-Leffler function defined by

$$
E_{\alpha}(x)=\sum_{k=0}^{\infty} \frac{x^{k}}{\Gamma(1+k \alpha)} .
$$

Substituting (17) along with (18) and setting the coefficient of $\varphi^{k}(\xi)$ equal to zero lead to a set of algebraic equations in terms of coefficients $a_{0}, a_{1}, a_{2}, b_{0}$ and $b_{2}$. Solving the algebraic set of equations by Maple gives the following cases:

Case 1: In this case, $A$ and $B$ are arbitrary while $C=0$. This case describes the Burgers equation. The coefficients of (19), using the Maple package, have the forms:

$$
\begin{gathered}
b_{0}=\text { arbitrary, } \quad b_{2}=1, \quad a_{0}=-v^{\alpha} / A, \\
a_{1}=-2 B / A, \quad a_{2}=0,
\end{gathered}
$$

where $A \neq 0$, the solutions of the STFKdV-Burgers equation using the fractional Riccate equation solutions (21) are as follows:

$$
\Phi_{11}(\xi)=-v^{\alpha} / A+(2 B / A) \sqrt{-b_{0}} \tanh _{\alpha}\left(\sqrt{-b_{0}} \xi\right), b_{0}<0,
$$

$\Phi_{12}(\xi)=-v^{\alpha} / A+(2 B / A) \sqrt{-b_{0}} \operatorname{coth}_{\alpha}\left(\sqrt{-b_{0}} \xi\right), b_{0}<0$,

$$
\Phi_{13}(\xi)=-v^{\alpha} / A-(2 B / A) \sqrt{b_{0}} \tan _{\alpha}\left(\sqrt{b_{0}} \xi\right), \quad b_{0}>0,
$$

$$
\Phi_{14}(\xi)=-v^{\alpha} / A+(2 B / A) \sqrt{b_{0}} \cot _{\alpha}\left(\sqrt{b_{0}} \xi\right), \quad b_{0}>0,
$$

$$
\Phi_{15}(\xi)=-v^{\alpha} / A+(2 B / A) \Gamma(1+\alpha) /\left(\xi^{\alpha}+\omega\right), \quad b_{0}=0,
$$

$$
\omega=\text { arbitrary constant. }
$$

Case 2: The second case has $A$ and $C$ arbitrary while $B=0$. This describes the KdV equation. The set of coefficients of the solution of (19) using the Maple package is

$$
\begin{gathered}
b_{0}=\text { arbitrary, } \mathrm{b}_{2}=1, \mathrm{a}_{0}=-\left(8 \mathrm{~b}_{0} \mathrm{C}+\mathrm{v}^{\alpha}\right) / \mathrm{A}, \\
a_{1}=0, \quad a_{2}=-12 C / A,
\end{gathered}
$$

where $A \neq 0$, these coefficients lead to the second set of solutions of the STFKdV-Burgers equation in the following forms

$$
\begin{aligned}
& \Phi_{21}(\xi)=-\left(8 b_{0} C+v^{\alpha}\right) / A \\
& +(12 C / A) b_{0}\left[\tanh _{\alpha}\left(\sqrt{-b_{0}} \xi\right)\right]^{2}, \quad b_{0}<0 \text {, } \\
& \Phi_{22}(\xi)=-\left(8 b_{0} C+v^{\alpha}\right) / A \\
& +(12 C / A) b_{0}\left[\operatorname{coth}_{\alpha}\left(\sqrt{-b_{0}} \xi\right)\right]^{2}, \quad b_{0}<0, \\
& \Phi_{23}(\xi)=-\left(8 b_{0} C+v^{\alpha}\right) / A \\
& -(12 C / A) b_{0}\left[\tan _{\alpha}\left(\sqrt{b_{0}} \xi\right)\right]^{2}, \quad b_{0}>0, \\
& \Phi_{24}(\xi)=-\left(8 b_{0} C+v^{\alpha}\right) / A \\
& -(12 C / A) b_{0}\left[\cot _{\alpha}\left(\sqrt{-b_{0}} \xi\right)\right]^{2}, \quad b_{0}>0, \\
& \Phi_{25}(\xi)=-\left(8 b_{0} C+v^{\alpha}\right) / A \\
& -(12 C / A)\left[\Gamma(1+\alpha) /\left(\xi^{\alpha}+\omega\right)\right]^{2}, \quad b_{0}=0, \\
& \omega=\text { constant } .
\end{aligned}
$$

Case 3: The third case has $A, B$ and $C$ arbitrary, which describe the KdV-Burgers equation. The set of coefficients of 


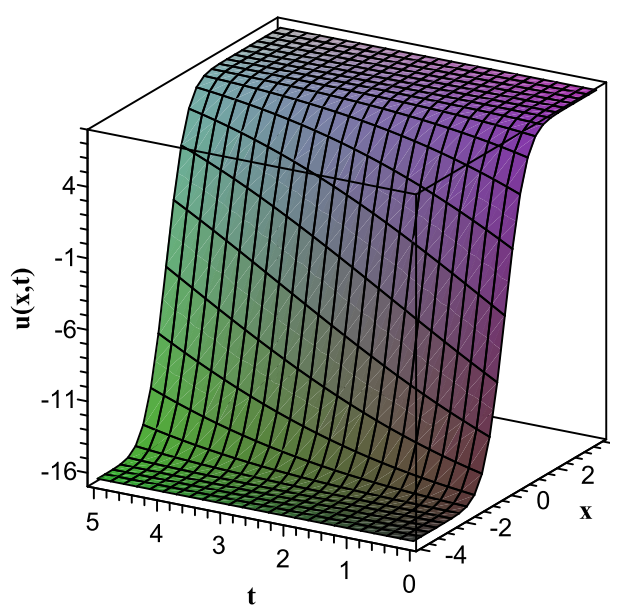

(a) 3-dimensions, $\alpha=0.5$

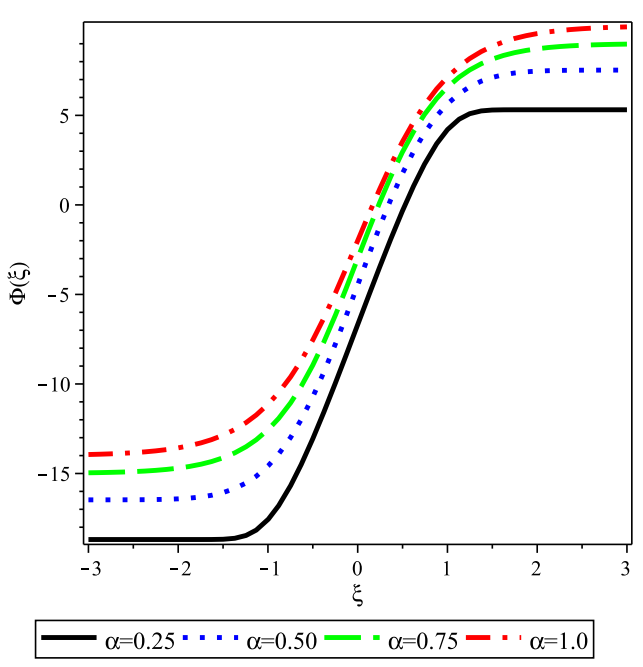

(b) 2-dimensions

Fig. 1. The solution $\Phi_{11}(\xi), \xi=x+v t$ for $A=0.1, B=0.6, C=0, b_{0}=-1, v=0.2$

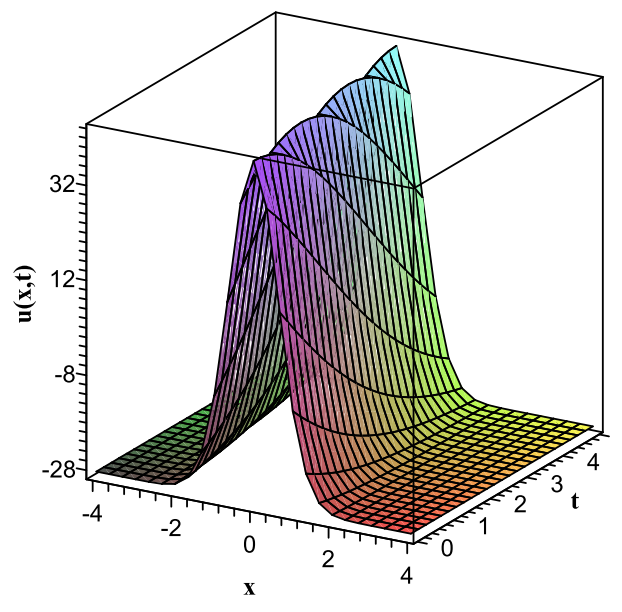

(a) 3-dimensions, $\alpha=0.5$

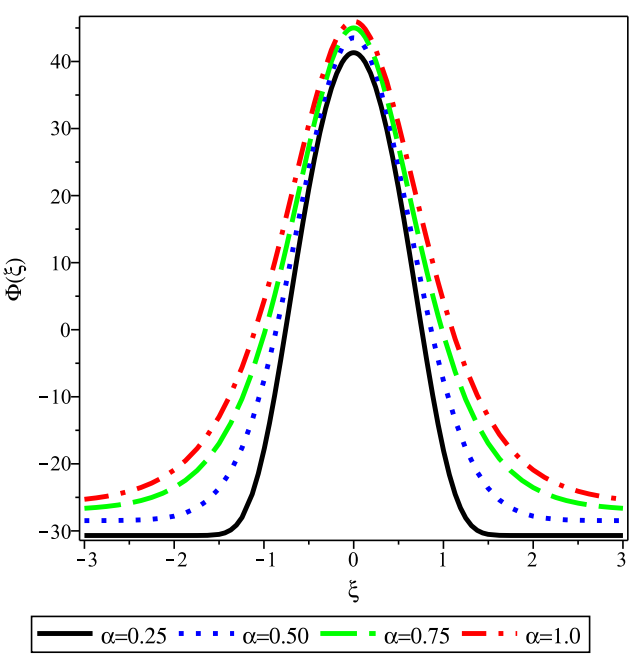

(b) 2-dimensions

Fig. 2. The solution $\Phi_{21}(\xi), \xi=x+v t$ for $A=0.1, B=0, C=0.6, b_{0}=-1, v=0.2$

the solution of STFKdV-Burgers equation is given by

$$
\begin{gathered}
b_{0}=-\frac{B^{2}}{100 C^{2}}, \quad b_{2}=1, \quad a_{0}=\frac{3 B^{2}-25 v^{\alpha} C}{25 A C}, \\
a_{1}=-\frac{12 B}{5 A}, \quad a_{2}=-\frac{12 C}{A},
\end{gathered}
$$

where $A C \neq 0$. As the coefficient $b_{0}$ is negative, therefore the solutions corresponding to this set of coefficients are two solutions only and represented by the following forms:

$$
\begin{aligned}
& \Phi_{31}(\xi)=\frac{3 B^{2}-25 v^{\alpha} C}{25 A C}+\frac{6 B^{2}}{25 A C} \tanh _{\alpha}\left(\frac{B}{10 C} \xi\right) \\
& -\frac{3 B^{2}}{25 A C}\left[\tanh _{\alpha}\left(\frac{B}{10 C} \xi\right)\right]^{2} . \\
& \Phi_{32}(\xi)=\frac{3 B^{2}-25 v^{\alpha} C}{25 A C}+\frac{6 B^{2}}{25 A C} \operatorname{coth}_{\alpha}\left(\frac{B}{10 C} \xi\right) \\
& -\frac{3 B^{2}}{25 A C}\left[\operatorname{coth}_{\alpha}\left(\frac{B}{10 C} \xi\right)\right]^{2} .
\end{aligned}
$$




\section{RESULTS AND DISCUSSION}

The real world physical processes are modeled by nonlinear partial differential equations. First order nonlinear partial differential equations model nonlinear waves and arise in gas dynamics, water waves, elastodynamics, chemical reactions, transport of pollutants, flood waves in rivers, chromatography, traffic flow, and a wide range of biological and ecological systems. Second order partial differential equations govern nonlinear diffusion processes, including thermodynamics, chemical reactions, dispersion of pollutants, and population dynamics, the simplest and best understood is Burger's equation. Third order partial differential equations arise in the study of dispersive wave motion as the $\mathrm{KdV}$ equation, including water waves, plasma waves, waves in elastic media. A generalization of these two equations ( $\mathrm{KdV}$ and Burgers) known as the KdV-B equation is very useful approximation to describe phenomena in fluid mechanics and plasma physics.

The real world physical processes can be better modeled by fractional differential equations rather by integer-order differential equations. The space-time fractional KdV-Burgers' equations are derived using Agrawal's technique [23-25] and

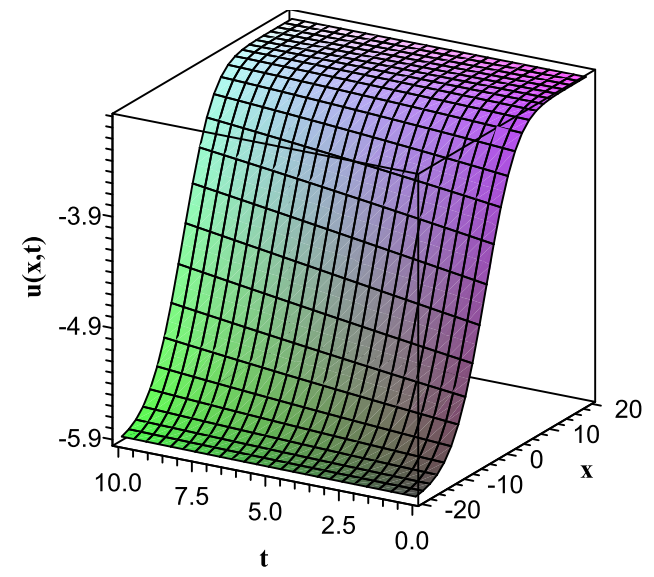

(a) 3-dimensions, $\alpha=0.5$

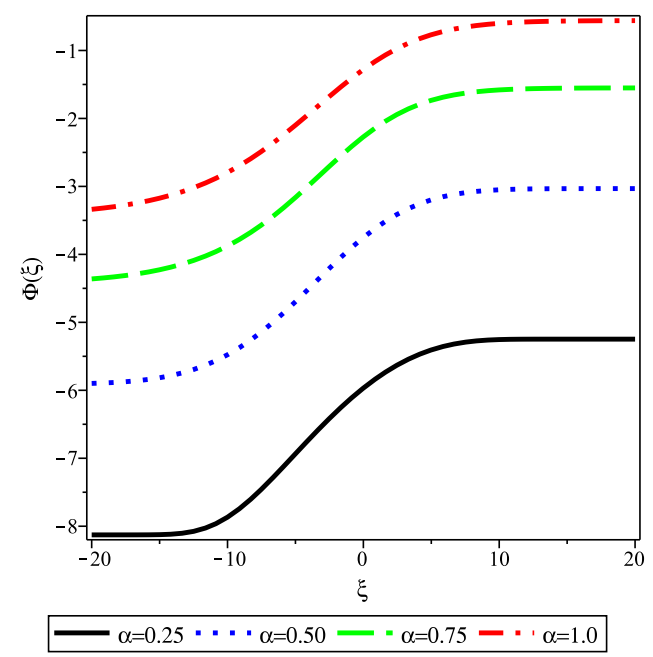

(b) 2-dimensions

Fig. 3. The solution $\Phi_{31}(\xi), \xi=x+v t$ for $A=0.1, B=0.6, C=0.6, b_{0}=-\frac{B^{2}}{100 C^{2}}, v=0.2$

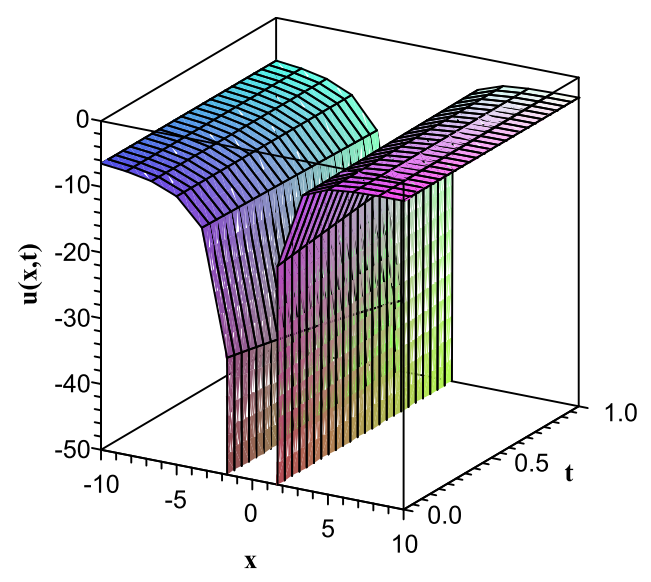

(a) 3-dimensions, $\alpha=0.5$

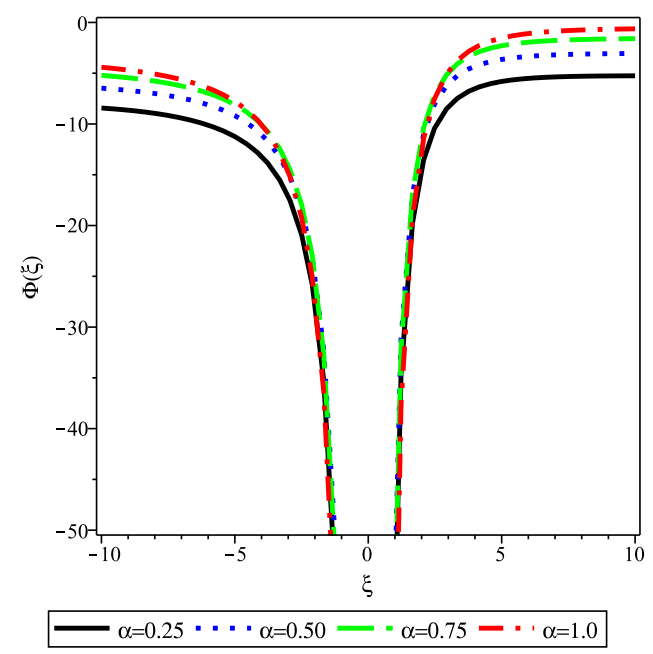

(b) 2-dimensions

Fig. 4. The solution $\Phi_{32}(\xi), \xi=x+v t$ for $A=0.1, B=0.6, C=0.6, b_{0}=-\frac{B^{2}}{100 C^{2}}, v=0.2$ 
the modified Riemann-Liouville derivative which were defined by Jumarie [12-15], and solved at different three cases. The first case takes the dissipation coefficient equal to zero, which tends to Burgers' equation, the second case takes the dispersion coefficient equal to zero, which tends to $\mathrm{KdV}$ equation, and the third case takes the dispersion and dissipation coefficient arbitrary that are not equal to zero, using the fractional sub-equation method $[34,35]$.

Fig. (1a) shows the solution $\Phi_{11}(\xi)$ in 3-dimensions where a shock wave formulated as the dispersion term in the KdV-Burgers equation equals to zero, and Fig (1b) shows the relation of $\Phi_{11}(\xi)$ and the position at different values of the fractional parameter $\alpha$, where as shown as $\alpha$ increase the amplitude of the shock wave increase. If the dissipation coefficient in the KdV-Burgers equation equals to zero this equation tends to $\mathrm{KdV}$ equation, where a soliton solution will be obtained as shown in Fig (2a) the relation between $\Phi_{21}(\xi)$, position, and time. Fig. (2b) shows the relation of $\Phi_{21}(\xi)$ and the position at different values of the fractional parameter $\alpha$, where a bell shape formulated which increased in the amplitude and width as the fractional parameter increased. Figures (3) and (4) show that at dispersion and dissipation coefficients are not equal to zero, where Fig (3) shows the relation between $\Phi_{31}(\xi)$ and the position in 3-dimensions and 2-dimensions at different values of fractional parameter, respectively. Fig. (4) shows the relation between $\Phi_{32}(\xi)$ and the position in 3-dimensions and 2-dimensions at different values of fractional parameter, respectively when the balance between nonlinear and dispersion effect is strong and can result in the formation of explosive waves which appear in different fields as tsunami.

\section{Acknowledgement}

All of the authors would like to pass their sincere acknowledgement to Prof. S.A. El-Wakil for his continuous guidance, encouragement, suggestions and review of their scientific work. Also, the authors have sincere thanks to the referees for their valuable comments which improve the paper.

\section{References}

[1] J.A. Tenreiro Machado, Fractional generalization of memristor and higher order elements, Communications in Nonlinear Science \& Numerical Simulation 18(2), 264-275 (2013).

[2] D. Baleanu, J.A. Tenreiro Machado, A.C.J. Luo, Editors, Fractional Dynamics and Control, Springer, New York, 2012.

[3] M.-A. Polo-Labarrios, G. Espinosa-Paredes, Application of the fractional neutron point kinetic equation: Start-up of a nuclear reactor, Annals of Nuclear Energy 46(1), 37-42 (2012).

[4] A. Kadem, D. Baleanu, Analytical method based on Walsh function combined with orthogonal polynomial for fractional transport equation, Communications in Nonlinear Science \& Numerical Simulation 15(3), 491-501 (2010).
[5] I.S. Jesus, J.A. Tenreiro Machado, R.S. Barbosa, Control of a heat diffusion system through a fractional order nonlinear algorithm, Computers \& Mathematics with Applications 59(5), 1687-1694 (2010).

[6] D. Baleanu, Z.B. Güvenç, J.A. Tenreiro Machado, Editors, New Trends in Nanotechnology and Fractional Calculus Applications, Springer, Dordrecht, 2010.

[7] F. Mainardi, Fractional Calculus and Waves in Linear Viscoelasticity: An Introduction to Mathematical Models, Imperial College Press, London, UK, 2010.

[8] D. Baleanu, K. Diethelm, E. Scalas, J.J. Trujillo, Fractional Calculus: Models and Numerical Methods, vol. 3 of series on Complexity, Nonlinearity and Chaos, World Scientific Publishing, Hackensack, NJ, 2012.

[9] A.A. Kilbas, H.M. Srivastava, J.J. Trujillo, Theory and Applications of Fractional Differential Equations, vol. 204 of North-Holland Mathematics Studies, Elsevier Science B. V., Amsterdam, 2006.

[10] I. Podlubny, Fractional Differential Equations, Academic Press, San Diego, 1999.

[11] S.G. Samko, A.A. Kilbas, O.I. Marichev, Fractional integrals and derivatives: theory and applications, Gordon and Breach Science Publishers, Amsterdam, 1993.

[12] G. Jumarie, On the fractional solution of the equation $f(x+y)=f(x) f(y)$ and its application to fractional Laplace's transform, Applied Mathematics and Computation 219(4), 1625-1643 (2012)

[13] G. Jumarie, An Approach to Differential Geometry of Fractional Order via Modified Riemann-Liouville Derivative, Acta Mathematica Sinica, English Series 28(9), 1741-1768 (2012).

[14] G. Jumarie, An approach via fractional analysis to nonlinearity induced by coarse-graining in space, Nonlinear Analysis: Real World Applications 11(1), 535-546 (2010).

[15] G. Jumarie, Table of some basic fractional calculus formulae derived from a modified Riemann-Liouville derivative for non-differentiable functions, Applied Mathematics Letters 22(3), 378-385 (2009).

[16] G.-w. Wang, X.-q. Liu, Y.-y. Zhang, Lie symmetry analysis to the time fractional generalized fifth-order KdV equation, Communications in Nonlinear Science \& Numerical Simulation 18(9), 2321-2326 (2013).

[17] H. Wang, T.-C. Xia, The fractional supertrace identity and its application to the super Jaulent-Miodek hierarchy, Communications in Nonlinear Science Numerical Simulation 18(10), 2859-2867 (2013).

[18] Z. Hammouch, T. Mekkaoui, Travelling-wave solutions for some fractional partial differential equation by means of generalized trigonometry functions, Int. J. Applied Mathematical Research 1(2), 206-212 (2012).

[19] R. Almeida, D.F.M. Torres, Fractional variational calculus for nondifferentiable functions, Computers \& Mathematics with Applications 61(10), 3097-3104 (2011).

[20] G.-c. Wu, A fractional variational iteration method for solving fractional nonlinear differential equations, Computers \& Mathematics with Applications 61(8), 2186-2190 (2011).

[21] F. Riewe, Nonconservative Lagrangian and Hamiltonian Mechanics, Physical Review E 53(2), 1890-1899 (1996).

[22] F. Riewe, Mechanics with Fractional Derivatives, Physical Review E 55(3), 3581-3592 (1997).

[23] O.P. Agrawal, Formulation of Euler-Lagrange Equations for Fractional Variational Problems, J. Mathematical Analysis \& Applications 272(1), 368-379 (2002).

[24] O.P. Agrawal, Fractional Variational Calculus in Terms of Riesz Fractional Derivatives, J. Physics A: Mathematical \& Theoretical 40, 6287-6303 (2007). 
[25] O.P. Agrawal, S.I. Muslih, D. Baleanu, Generalized variational calculus in terms of multi-parameters fractional derivatives, Communications in Nonlinear Science \& Numerical Simulation 16(12), 4756-4767 (2011).

[26] M.A.E. Herzallah, S.I. Muslih, D. Baleanu, E.M. Rabei, Hamilton-Jacobi and fractional like action with time scaling, Nonlinear Dynamics 66(4), 549-555 (2011).

[27] M.A.E. Herzallah, D. Baleanu, Fractional Euler-Lagrange equations revisited, Nonlinear Dynamics 69(3), 977-982 (2012).

[28] S.A. El-Wakil, E.M. Abulwafa, M.A. Zahran, A.A. Mahmoud, Formulation of Some Fractional Evolution Equations used in Mathematical Physics, Nonlinear Science Letters A 2(1), 37-46 (2011).

[29] S.A. El-Wakil, E.M. Abulwafa, E.K. El-Shewy, A.A. Mahmoud, Time-fractional study of electron acoustic solitary waves in plasma of cold electron and two isothermal ions, J. Plasma Physics 78(6), 641-649 (2012).

[30] A.M.A. El-Sayed, S.H. Behiry, W.E. Raslan, A numerical algorithm for the solution of an intermediate fractional advection dispersion equation, Communications in Nonlinear Science \& Numerical Simulation 15(5): 1253-1258 (2010).

[31] S. Momani, Z. Odibat, Numerical comparison of methods for solving linear differential equation of fractional order, Chaos, Solitons \& Fractals 31(5), 1248-1255 (2007).
[32] J.H. He, Homotopy perturbation technique, Computer Methods in Applied Mechanics \& Engineering 178(3-4), 257-262 (1999).

[33] E.M. Abulwafa, M.A. Abdou, A.A. Mahmoud, The Variational-Iteration Method to Solve the Nonlinear Boltzmann Equation, Zeitschrift für Naturforschung A 63a(34), 131-139 (2008).

[34] S. Zhang, H.-Q. Zhang, Fractional sub-equation method and its applications to nonlinear fractional PDEs, Physics Letters A 375(7), 1069-1073 (2011).

[35] S. Guo, L. Mei, Y. Li, Y. Sun, The improved fractional subequation method and its applications to the space-time fractional differential equations in fluid mechanics, Physics Letters A 376(4), 407-411 (2012).

[36] J.-H. He, Semi-inverse Method of Establishing Generalized Variational Principles for Fluid Mechanics with Emphasis on Turbo-Machinery Aerodynamics, Int. J. Turbo Jet-Engines 14(1), 23-28 (1997).

[37] J.-H. He, Variational Principles for Some Nonlinear Partial Differential Equations with Variable Coefficients, Chaos, Solitons \& Fractals 19(4), 847-851 (2004).

[38] S. Zhang, Q.A. Zong, D. Liu, Q. Gao, A Generalized ExpFunction Method for Fractional Riccati Differential Equations, Communications in Fractional Calculus 1(1), 48-51 (2010).
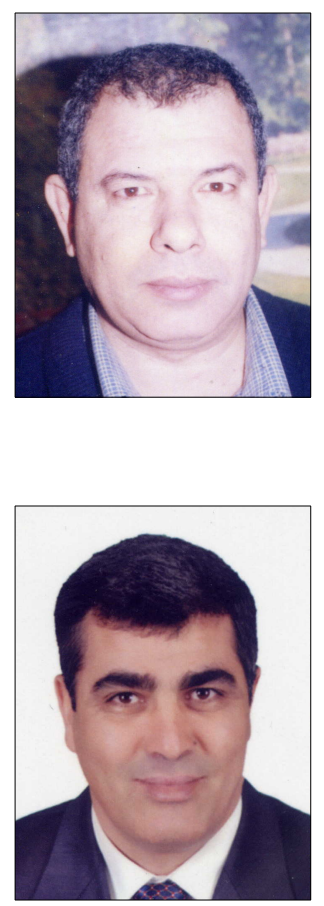

Essam M. Abulwafa, born on $31^{\text {st }}$ December 1954, received his BSc in Physics (1976), MSc (1982) and $\mathrm{PhD}$ (1989) in theoretical nuclear physics from Mansoura University, Mansoura, Egypt. He has been Professor of Theoretical Physics at the Physics Department, Faculty of Science, Mansoura University, Mansoura, Egypt, since December 1999. e-mail: abulwafa@mans.edu.eg.
Ahmed M. Elgarayhi, born on $12^{\text {th }}$ March 1963, received his BSc in Physics (1985), MSc (1989) and PhD (1994) in theoretical physics from Mansoura University, Mansoura, Egypt. He has been Professor of theoretical physics at the Physics Department, Faculty of Science, Mansoura University, Mansoura, Egypt, since March 2006. e-mail: elgarayhi@mans.edu.eg. 


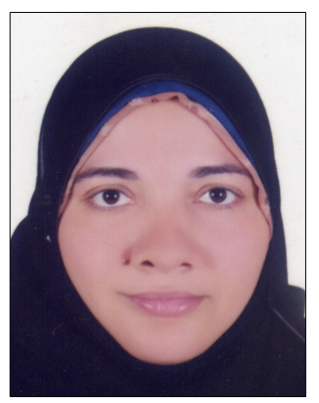

Abeer A. Mahmoud received her BSc in Physics (2000), MSc (2006) and PhD (2011) in theoretical physics from Mansoura University, Mansoura, Egypt. She has been Lecturer of theoretical physics at the Physics Department, Faculty of Science, Mansoura University, Mansoura, Egypt, since June 2011. e-mail: abeer_wd@mans.edu.eg.

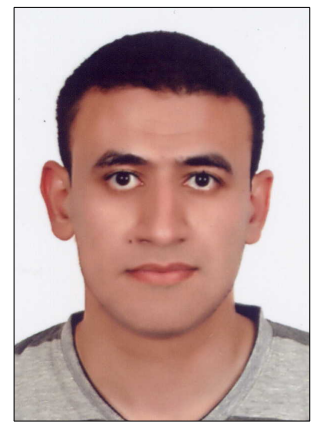

Ashraf M. Tawfik, born on $4^{\text {th }}$ December 1989, received his BSc in Physics (2010) from Mansoura University, Mansoura, Egypt. He has been Demonstrator of physics in Physics Department, Faculty of Science, Mansoura University, Mansoura, Egypt, since 2010. e-mail: ashroof_tawfik@ mans.edu.eg. 